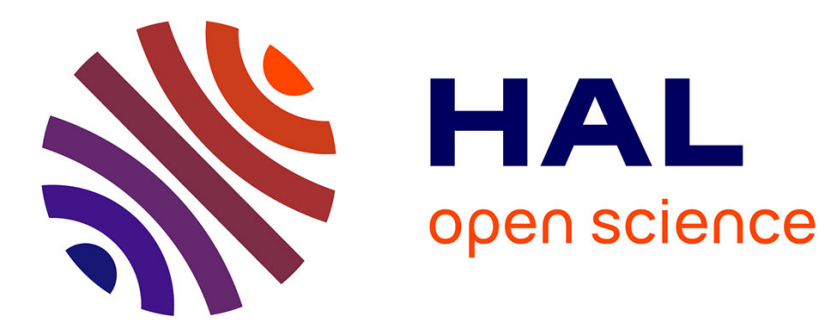

\title{
Impact du champignon parasite Phellinus robustus et des cavités nidifiables sur la croissance des Chênes sessile et pédonculé.
}

\author{
Elvire Hatsch, Jean-Luc Dupouey, B. Dubreuil, J. Guillaud
}

\section{To cite this version:}

Elvire Hatsch, Jean-Luc Dupouey, B. Dubreuil, J. Guillaud. Impact du champignon parasite Phellinus robustus et des cavités nidifiables sur la croissance des Chênes sessile et pédonculé.. Revue forestière française, 1999, 51 (4), pp.511-521. 10.4267/2042/5459 . hal-03443414

HAL Id: hal-03443414

https://hal.science/hal-03443414

Submitted on 23 Nov 2021

HAL is a multi-disciplinary open access archive for the deposit and dissemination of scientific research documents, whether they are published or not. The documents may come from teaching and research institutions in France or abroad, or from public or private research centers.
L'archive ouverte pluridisciplinaire HAL, est destinée au dépôt et à la diffusion de documents scientifiques de niveau recherche, publiés ou non, émanant des établissements d'enseignement et de recherche français ou étrangers, des laboratoires publics ou privés. 


\title{
IMPACT DU CHAMPIGNON PARASITE PHELLINUS ROBUSTUS ET DES CAVITÉS NIDIFIABLES SUR LA CROISSANCE DES CHÊNES SESSILE ET PÉDONCULÉ
}

\author{
Elvire HATSCH - J.-L. DUPOUEY \\ B. DUBREUIL (†) - J. GUILLAUD
}

La présence des arbres à cavités nidifiables augmente la diversité écologique de la forêt en favorisant l'installation d'oiseaux et de mammifères divers. L'existence de ces cavités est souvent associée à la présence d'un champignon parasite, Phellinus robustus Karst. Ce champignon est fréquemment situé à la base du houppier ou dans le houppier et se développe sur le tronc ou sur les branches (vivantes ou sèches). II s'installe à la faveur d'une blessure ou dans les cicatrices de branches qui sont tombées. Le mycélium se développe dans un premier temps à l'intérieur de l'arbre et le carpophore ne devient visible qu'au bout de plusieurs années (Dubreuil, 1997b). II attaque presque exclusivement le Chêne et provoque une pourriture fibreuse dans laquelle les pics vont creuser des cavités qui leur serviront de lieu de nidification.

Ces cavités pourront également être occupées par de nombreuses autres espèces, y compris des mammifères comme la martre, car les pics ne nichent qu'une seule année au même endroit (Pautz, 1998). Les oiseaux les plus fréquents qui vont nicher dans ces cavités sont des pics, des mésanges, des étourneaux, la sittelle et le gobe-mouches à collier (Pautz et Dubreuil, 1997).

Ces arbres sont en général rapidement éliminés de la forêt lors des martelages, de peur que le bois ne se déprécie ou que d'autres arbres soient atteints. La présence du champignon parasite et de cavités peut être également le reflet d'un affaiblissement ou d'un dépérissement des arbres. Cependant, maintenir ces arbres dans le peuplement permet de garder une biodiversité importante au niveau de la faune.

Une étude a été initiée en Moselle par l'Office national des Forêts ; elle a pour objectif de déterminer la genèse et l'évolution de la cavité et de mesurer l'impact économique de ce phénomène.

Dans le cadre de cette étude, nous avons effectué une analyse dendroécologique afin de déterminer les relations entre, d'une part, la présence de la cavité et du champignon parasite et, d'autre part, la croissance des chênes. II s'agit notamment de vérifier si l'altération du bois peut provoquer des pertes de croissance significatives au niveau de la bille de pied. 


\section{MATÉRIELS ET MÉTHODES}

Pour cette étude, nous avons étudié 150 chênes, sessile et pédonculé (Quercus petraea (Matt.) Lieb. et $Q$. robur L.), issus de trois parcelles de la forêt domaniale de Hémilly en Moselle. Cette forêt est située sur le plateau lorrain à $70 \mathrm{~km}$ au nord-est de Nancy et $30 \mathrm{~km}$ à l'est de Metz. Pour l'ensemble des arbres, la moyenne d'âge est de 154 ans et le diamètre moyen de $55 \mathrm{~cm}$. 144 arbres ont été échantillonnés dans les parcelles 14 et 15 qui sont voisines et sont d'anciens taillis-sousfutaie mis en conversion vers 1880. Les arbres y ont des âges et des diamètres peu différents (respectivement 153 et 160 ans et 53 et $59 \mathrm{~cm}$ de diamètre). Les six autres, tous sessiles, sont situés dans la parcelle 26 , jeune futaie régulière dont l'âge moyen est de 86 ans pour un diamètre moyen de $36 \mathrm{~cm}$.

Ces parcelles se subdivisent en trois types de stations. La station A, bien drainée, est caractérisée par un sol brun lessivé avec une épaisseur de limon supérieure à $30 \mathrm{~cm}$ et se situe en sommet de pente. La station C, mal drainée et à hydromorphie temporaire, est située en bas de pente. Cette station est caractérisée par un pélosol pseudogley dont l'épaisseur de limon n'excède pas $25 \mathrm{~cm}$. La station $\mathrm{B}$, intermédiaire, est caractérisée par un pélosol ou pélosol brunifié relativement bien drainé et recouvert d'une épaisseur de limon de moins de $30 \mathrm{~cm}$.

Les deux espèces de Chênes sont en mélange mais leur fréquence varie selon la station (tableau I, ci-dessous), en accord avec les différences autécologiques qui les distinguent.

Tableau I Moyennes des âges, des diamètres et des largeurs de cerne à 1,30 m par espèce et par type de station

\begin{tabular}{|l|c|c|c|c|c|c|c|c|c|c|c|c|c|}
\hline & \multicolumn{3}{|c|}{ Âge (ans) } & \multicolumn{4}{c|}{ Diamètre (cm) } & \multicolumn{2}{c|}{$\begin{array}{c}\text { Largeur moyenne } \\
\text { de cerne (mm) }\end{array}$} & \multicolumn{5}{c|}{ Effectif } \\
\hline Station & A & B & C & A & B & C & A & B & C & A & B & C & total \\
\hline Pédonculé & 177 & 156 & 153 & 64 & 51 & 63 & 1,54 & 1,43 & 1,83 & 9 & 23 & 44 & 76 \\
Sessile & 154 & 138 & 147 & 51 & 43 & 52 & 1,46 & 1,34 & 1,50 & 60 & 12 & 2 & 74 \\
\hline Ensemble & 157 & 150 & 153 & 53 & 48 & 63 & 1,47 & 1,40 & 1,82 & 69 & 35 & 46 & 150 \\
\hline
\end{tabular}

Les arbres ont été choisis par couples, parmi les dominants et les codominants. Pour chaque arbre présentant un défaut (cavité ou champignon), nous avons échantillonné le complémentaire sain le plus proche de même espèce et de même classe de diamètre.

La moitié des arbres étudiés présente donc un défaut. Sur ces 75 arbres, 39 possèdent des carpophores et des cavités associés, 10 n'ont pas de cavité mais au moins un carpophore visible et 26 n'ont pas de carpophore visible mais au moins une cavité nidifiable. Si aucun carpophore n'est visible mais qu'il existe une cavité, nous pouvons supposer que le champignon parasite est présent à l'état de mycélium qui a ramolli le bois car le pic n'arrive pas à forer dans du bois sain (Pautz, 1998).

Par convention, dans les tableaux et les analyses qui suivent, nous avons regroupé les chênes avec cavités ou champignons sous la dénomination "chênes parasités".

Pour chaque arbre, nous disposons de sa hauteur et de son diamètre à 1,30 m. Pour les arbres parasités, des informations concernant le champignon (nombre de carpophores, position, longueur de la zone atteinte) et les cavités (nombre, espèce nicheuse, position) sont notées tous les ans depuis 1993 (Dubreuil, 1997a et b). L'espèce de Chêne est identifiée sur des critères morphologiques à partir de feuilles ramassées au pied des arbres (Dupouey et Badeau, 1993). 
En décembre 1997, nous avons prélevé une carotte à 1,30 m du côté Sud de chaque arbre, sauf défaut particulier. Nous avons mesuré la largeur de chaque cerne à l'aide d'une table à digitaliser associée à un système informatique (précision de 0,01 mm). Nous disposons donc de la largeur des cernes de chaque arbre, de leur âge par rapport à la moelle et de leur année de formation. Nous avons effectué des tests t appariés (comparaison de moyennes adaptée à la méthode des couples) de la différence de largeur de cerne entre arbres parasités et témoins, pour mettre en évidence d'éventuelles relations entre la présence du champignon ou de la cavité et la croissance des arbres. Nous avons également étudié les courbes de croissance au cours du temps des arbres parasités et des arbres témoins.

\section{RÉSULTATS}

\section{Différences d'âge et de croissance en diamètre entre les arbres parasités et les arbres sains}

En prenant tous les arbres en compte, nous observons une différence significative au seuil de $5 \%$ entre l'âge des chênes présentant un défaut (altération liée à la présence du champignon ou de cavités nidifiables) et celui des témoins. Les arbres parasités ont en moyenne 8 ans de plus que les arbres témoins.

En réalité, selon l'espèce et la station, les résultats sont plus variables. Pour le Chêne sessile, quelle que soit la station, les arbres parasités sont plus âgés que les arbres témoins (tableau II, cidessous). Les Chênes pédonculés des stations $A$ et $B$ montrent le même comportement. Par contre, sur la station $\mathrm{C}$, les Chênes pédonculés parasités sont moins âgés que les témoins mais la différence d'âge n'est pas significative (figure 1, p. 514 et tableau II, ci-dessous).

Les différences d'âge observées ne sont pas dues à des écarts de diamètre, puisque les arbres ont été groupés par couples à diamètre identique. Hormis les chênes pédonculés de la station $\mathrm{C}$, les arbres parasités ont donc une croissance radiale moyenne inférieure à celle des arbres témoins.

Tableau II

Test apparié de comparaison des âges et des diamètres entre les arbres parasités et les arbres témoins

Le tableau donne l'écart moyen entre arbres parasités et témoins pour l'âge (en années) et pour le diamètre (en cm) à $1,30 \mathrm{~m}$, différenciés par espèce et par station. L'effectif correspond au nombre de couples d'arbres (arbre parasité et témoin associé). Les tests t ne sont pas effectués pour les effectifs inférieurs à 5 couples d'arbres.

\begin{tabular}{|c|c|c|c|c|c|}
\hline & $\begin{array}{c}\text { Effectif } \\
(\mathrm{n}=75)\end{array}$ & $\begin{array}{c}\text { Écart moyen } \\
\text { pour l'âge }\end{array}$ & $\begin{array}{c}\text { Test t } \\
\text { pour l'âge }\end{array}$ & $\begin{array}{c}\text { Écart moyen } \\
\text { pour le diamètre }\end{array}$ & $\begin{array}{c}\text { Test t } \\
\text { pour le diamètre }\end{array}$ \\
\hline $\begin{array}{c}\text { Pédonculé } \\
\text { station A }\end{array}$ & 4 & 22,5 & $l$ & -2 & $I$ \\
\hline $\begin{array}{c}\text { Pédonculé } \\
\text { station B }\end{array}$ & 12 & 10,1 & $*$ & $-0,6$ & n.s. \\
\hline $\begin{array}{c}\text { Pédonculé } \\
\text { station C }\end{array}$ & 22 & $-4,7$ & n.s. & 0,4 & n.s. \\
\hline $\begin{array}{c}\text { Sessile } \\
\text { station A }\end{array}$ & 30 & 13,1 & $* *$ & -1 & n.s. \\
\hline $\begin{array}{c}\text { Sessile } \\
\text { station B }\end{array}$ & 6 & 21,2 & $*$ & 1 & n.s. \\
\hline $\begin{array}{c}\text { Sessile } \\
\text { station C }\end{array}$ & 1 & 1 & $l$ & -1 & $I$ \\
\hline
\end{tabular}

Écart significativement différent de 0 au seuil de $1 \%\left(^{* *}\right), 5 \%\left(^{*}\right)$ ou non significativement différent de 0 (n.s.). 


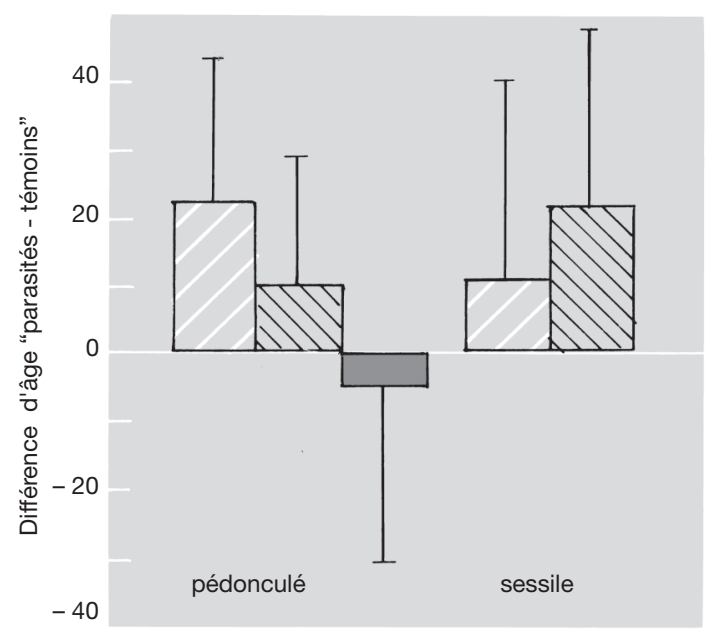

Figure 1

DIFFÉRENCE D'ÂGE MOYEN ENTRE LES ARBRES PARASITÉS ET LES ARBRES TÉMOINS, EN FONCTION DE L'ESPĖCE ET DE LA STATION

Les barres représentent les écarts-types.

Les Chênes sessiles de la station C

ne sont pas représentés car nous n'avons qu'un seul couple d'arbres.

Figure 2

ÉVOLUTION DU DIAMÈTRE EN FONCTION

DE L'ÂGE, POUR LES ARBRES

PARASITÉS ET LES ARBRES TÉMOINS

Les deux espèces sont confondues.

À partir de la mesure des largeurs de cerne, nous avons reconstitué l'évolution du diamètre de chaque arbre en fonction de l'âge. La figure 2 montre que les pertes de croissance des arbres parasités sont relativement faibles. Elles sont de l'ordre de $3,8 \mathrm{~cm}$ à l'âge de 150 ans, soit $8 \%$ de perte de diamètre par rapport aux arbres témoins.

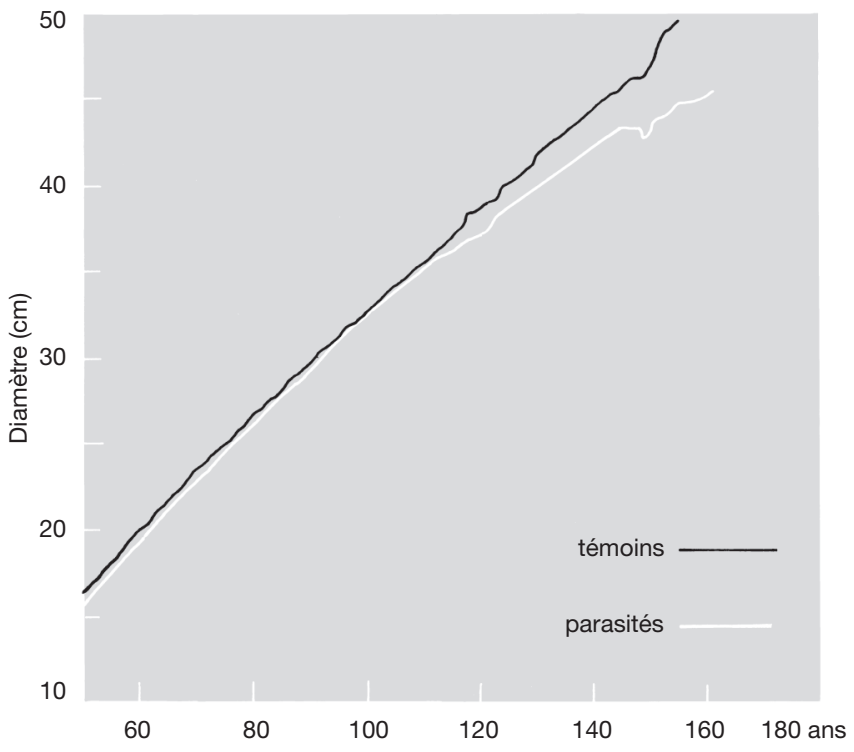

\section{Différences de largeur moyenne de cerne à 1,30 m}

La largeur moyenne de cerne à 1,30 m diffère peu entre arbres parasités et témoins, tous arbres et toutes parcelles confondus (test $t$ non significatif).

Si nous séparons les deux espèces, les Chênes pédonculés parasités présentent une différence de largeur moyenne de cerne avec les témoins quasiment nulle $(-0,003 \mathrm{~mm})$, alors qu'elle est de $-0,1 \mathrm{~mm}$ pour les Chênes sessiles.

Lorsque nous différencions par espèce et par station, l'écart entre la largeur moyenne de cerne des arbres parasités et celle des arbres témoins est toujours négatif sauf pour les Chênes pédonculés sur la station C (figure 3a, p. 515 et tableau III, p. 515). Cependant, ces différences ne sont jamais significatives. 
Le champignon parasite n'a pas toujours été présent dans l'arbre et nous ne connaissons pas la date de son installation. Nous avons donc aussi étudié les différences de croissance sur une période plus courte, de 1988 à 1997, de façon à nous placer de façon sûre dans la période d'infestation.

Sur ces dix dernières années, les arbres parasités montrent, toutes espèces et stations confondues, des largeurs moyennes de cerne nettement inférieures à celles des arbres témoins, de façon significative (figure $3 \mathrm{~b}$ et tableau III, ci-dessous). Ces différences sont significatives pour le Chêne

Figure 3 DIFFÉRENCE DE LARGEUR MOYENNE DE CERNE ENTRE ARBRES PARASITÉS ET TÉMOINS, EN FONCTION DE L'ESPĖCE ET DE LA STATION
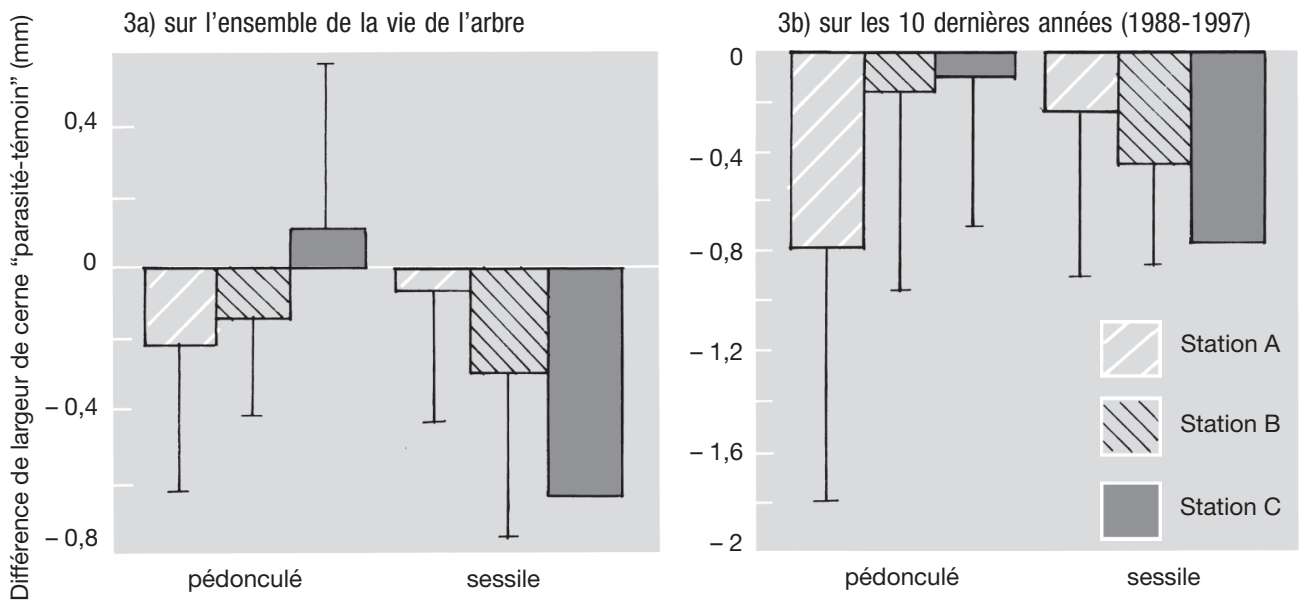

Tableau III

Comparaison des largeurs moyennes de cerne

sur l'ensemble de la vie des arbres et sur les 10 dernières années (1987-1997) entre les arbres parasités et les arbres témoins.

L'effectif correspond au nombre de couples d'arbres (arbre parasité et témoin associé)

\begin{tabular}{|c|c|c|c|c|c|}
\hline & Effectif $(n=75)$ & $\begin{array}{c}\text { Écart moyen } \\
\text { pour la largeur } \\
\text { moyenne } \\
\text { de cerne }\end{array}$ & Test t apparié & $\begin{array}{c}\text { Écart moyen } \\
\text { pour la largeur } \\
\text { moyenne } \\
\text { de cerne sur les } \\
10 \text { dernières } \\
\text { années }\end{array}$ & Test t apparié \\
\hline $\begin{array}{c}\text { Pédonculé } \\
\text { station A }\end{array}$ & 4 & $-0,21$ & $l$ & $-0,78$ & I \\
\hline $\begin{array}{c}\text { Pédonculé } \\
\text { station B }\end{array}$ & 12 & $-0,14$ & n.s. & $-0,15$ & n.s. \\
\hline $\begin{array}{c}\text { Pédonculé } \\
\text { station C }\end{array}$ & 22 & 0,11 & n.s. & $-0,08$ & n.s. \\
\hline $\begin{array}{c}\text { Sessile } \\
\text { station A }\end{array}$ & 30 & $-0,07$ & n.s. & $-0,30$ & * \\
\hline $\begin{array}{c}\text { Sessile } \\
\text { station B }\end{array}$ & 6 & $-0,30$ & n.s. & $-0,47$ & $/$ \\
\hline $\begin{array}{c}\text { Sessile } \\
\text { station C }\end{array}$ & 1 & $-0,65$ & $l$ & $-0,75$ & \\
\hline
\end{tabular}

Significativité des tests : cf. tableau II, p. 513. 
Elvire HATSCH - J.-L. DUPOUEY - B. DUBREUIL (†) - J. GUILLAUD

Figure 4 ÉVOLUTION DE LA LARGEUR DE CERNE POUR LES ARBRES PARASITÉS ET LES ARBRES TÉMOINS

Les courbes démarrent en 1875, date à laquelle les effectifs deviennent suffisants (30 couples)

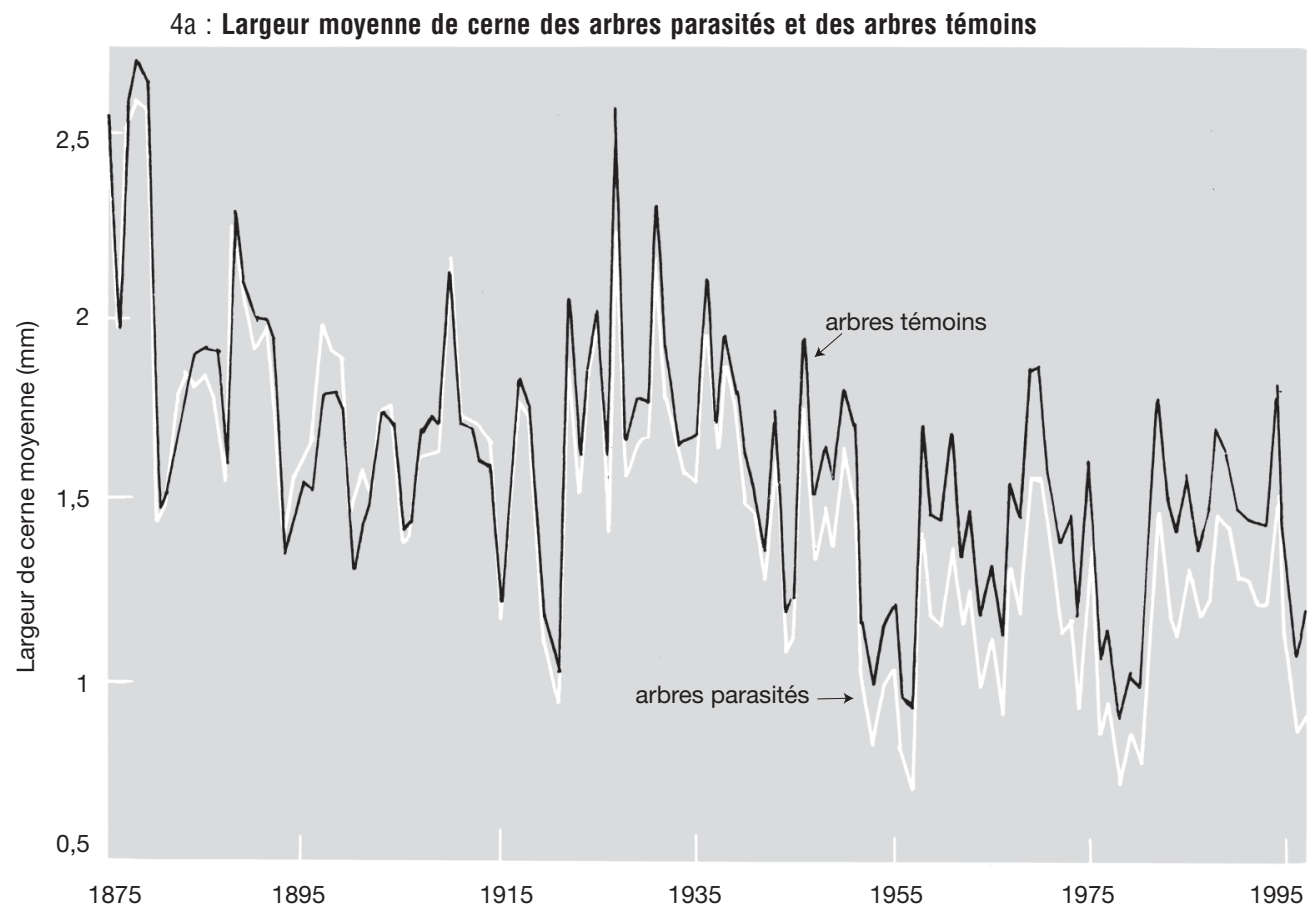

4b : Différence de largeur de cerne entre arbres parasités et témoins

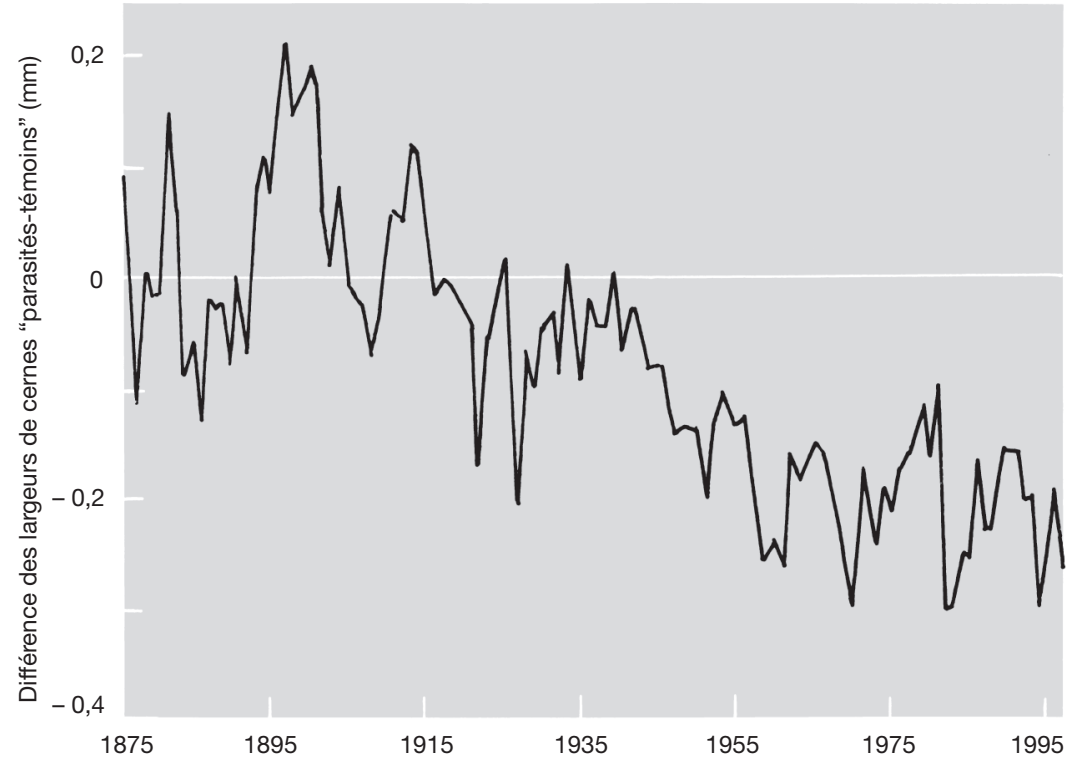


sessile sur les stations A et B (la station C n'est pas prise en compte par manque d'arbres). Par contre, elles ne le sont plus pour le Chêne pédonculé, mais les différences restent négatives quelle que soit la station, contrairement à ce que nous avions observé pour la croissance moyenne sur l'ensemble de la vie de l'arbre. Les Chênes pédonculés de la station C montrent là encore l'écart le moins négatif $(-0,08 \mathrm{~mm})$.

L'écart de croissance moyenne entre arbres parasités et témoins est, pour chacune des deux espèces, d'autant plus faible en valeur absolue que l'espèce est située en station favorable (figure 3b, p. 515).

\section{Historique de la croissance radiale au cours du siècle écoulé}

Les largeurs moyennes de cerne des arbres parasités et des arbres témoins sont bien synchronisées jusqu'aux années 1920-1925, puis un décalage apparaît entre les courbes (figure 4a et b, p. 516). Ce décalage se creuse ensuite progressivement, jusqu'à atteindre $0,25 \mathrm{~mm} / \mathrm{an}$ dans les dernières années.

II est intéressant de noter que, pour tous les couples d'arbres, l'écart entre parasité et témoin se creuse au cours des années qui suivent les périodes de faible croissance. Ainsi, les pertes de croissance de la seconde moitié des années 1970 sont suivies d'une amplification de la différence de croissance entre arbres parasités et témoins dans la première moitié des années 1980. On observe le même phénomène à la fin des années 1950 : les arbres aujourd'hui parasités ne retrouvent pas, à la suite de ces années défavorables, un niveau de croissance équivalent à celui qu'ils avaient auparavant, contrairement aux arbres témoins.

Figure 5

\section{ÉVOLUTION DE LA DIFFÉRENCE DES LARGEURS DE CERNE ENTRE CHÊNES PÉDONCULÉS PARASITÉS ET TÉMOINS au cours du temps, différenciée selon la station}

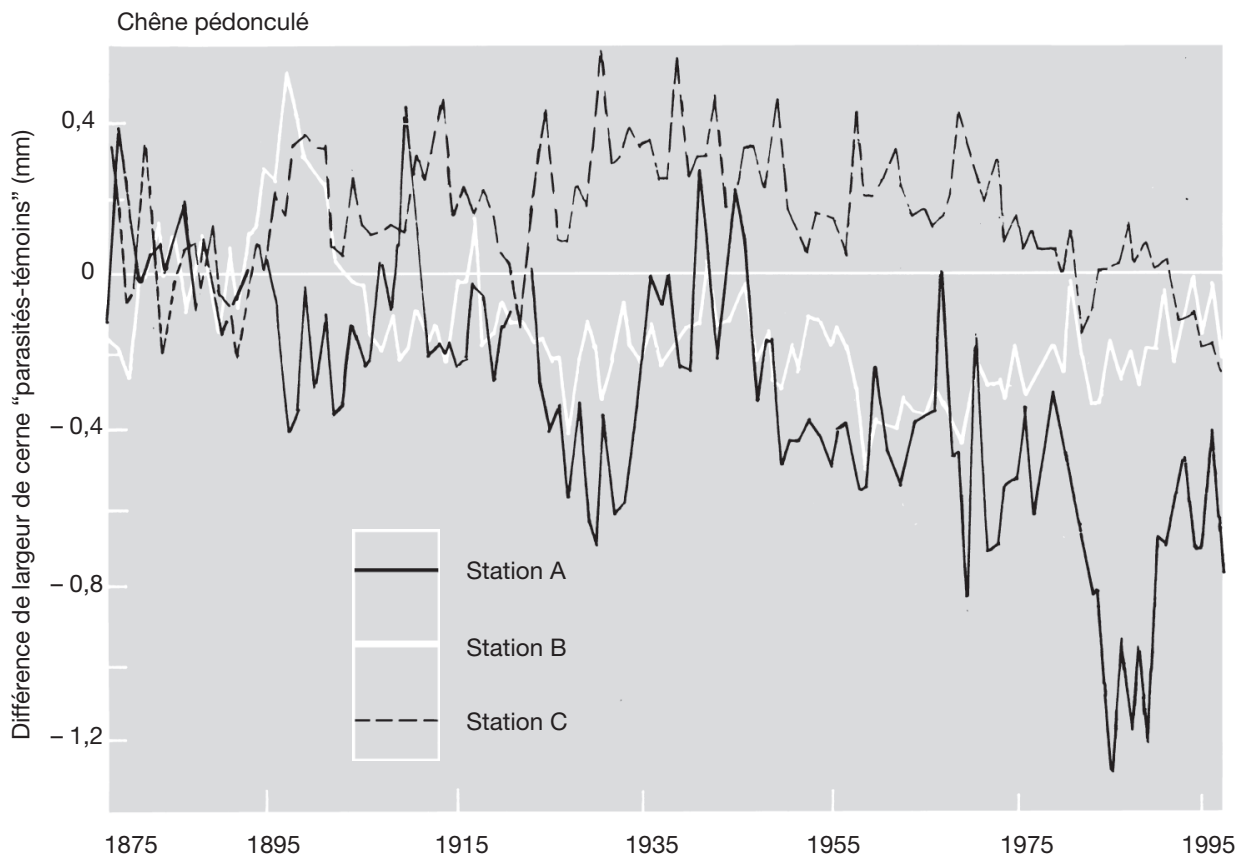


Comme précédemment, les différences entre arbres parasités et témoins varient selon l'espèce et le type de station. Là encore, l'écart apparaît d'autant plus précocement, et son ampleur est d'autant plus forte que l'espèce est située en station défavorable : pédonculé en station $A$, ou sessile en station B (figure 5, p. 517).

Le pédonculé en station $\mathrm{C}$ a un comportement particulier, puisque les arbres parasités semblent y pousser mieux que les arbres témoins, du moins jusque dans les années 1970.

Sur les stations $A$ et $B$, la différence de croissance entre chênes pédonculés parasités et témoins devient négative vers les années 1920 et le reste de façon définitive (figure 5, p. 517). Sur la station A, la réduction de croissance subie en 1920 se maintient et va même s'accentuer au cours du temps. Cependant, les effectifs sont faibles. Sur la station B, la perte de croissance des arbres parasités semble moins forte que sur la station A. Les arbres parasités gardent une croissance plus faible que celle des arbres témoins jusqu'à nos jours mais cette différence de croissance semble s'estomper progressivement.

La croissance des chênes pédonculés parasités sur la station $C$ est supérieure à celle des témoins jusqu'en 1970, malgré un court épisode de diminution en 1920. Elle s'effondre ensuite jusqu'à devenir inférieure à celle des témoins, à la suite de la crise de croissance des années 1976-1980. II semble donc que, sur leur station optimale, les chênes pédonculés parasités soient capables de récupérer un niveau de croissance élevé après les épisodes de faible croissance. II serait intéressant de suivre ces arbres plus longtemps afin de vérifier s'ils sont encore capables de récupérer à la suite de l'épisode actuel.

Figure 6 ÉVOLUTION DE LA DIFFÉRENCE DES LARGEURS DE CERNE ENTRE CHÊNES SESSILES PARASITÉS ET TÉMOINS au cours du temps, différenciée selon la station

Les chênes sessiles de la station C ne sont pas figurés car ils ne sont représentés que par un seul couple d'arbre.

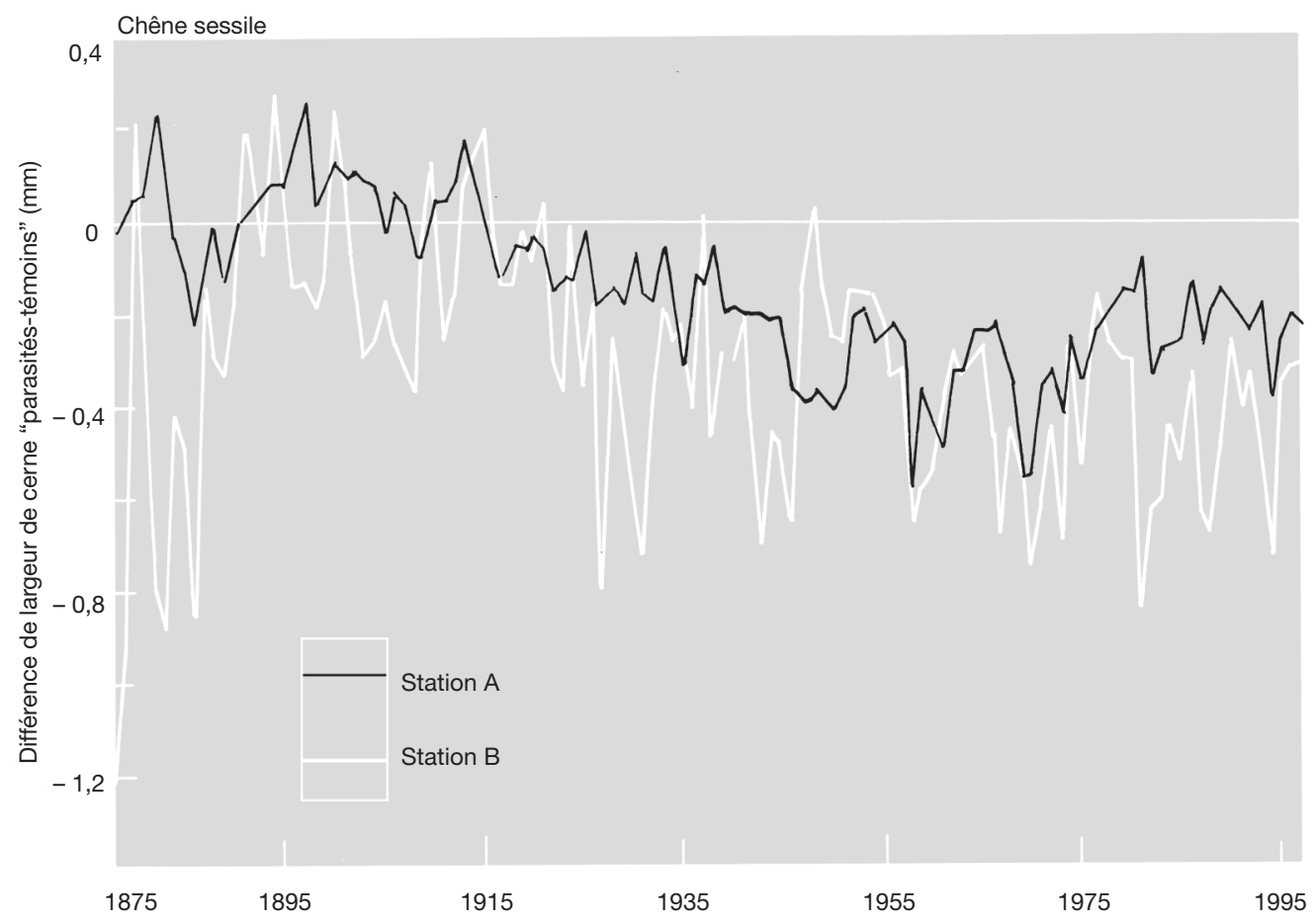


Le Chêne sessile se comporte de la même manière quelle que soit la station (figure 6, p. 518). Sur les deux stations, les arbres parasités ont subi une forte réduction de croissance à partir des années 1920 et ces arbres ne retrouvent plus un niveau de croissance similaire à celui des témoins. L'écart entre les courbes s'accentue progressivement pour les arbres de la station B qui semblent plus touchés que ceux de la station $A$.

\section{DISCUSSION ET CONCLUSIONS}

II est difficile de séparer le rôle respectif de la présence du champignon et des cavités et l'état de faiblesse de l'arbre lié à d'autres causes préexistantes (âge, variabilité génétique, micro-environnement ou concurrence) dans les pertes de croissance progressives des chênes parasités.

Malgré une longue période initiale au cours de laquelle il n'y a pas de différence de croissance entre l'arbre aujourd'hui sain et le témoin parasité associé, les écarts entre les deux groupes apparaissent très anciennement, dès les années 1920. L'année 1921 est connue comme l'une des sécheresses les plus importantes du siècle (Schérer et Fleisch, 1997). Ces différences s'amplifient à la suite de nouveaux événements climatiques extrêmes, sécheresses (1976) ou gels (1956). Après chacun de ces événements, les arbres témoins récupèrent leur niveau de croissance antérieur dans les années qui suivent, alors que les arbres aujourd'hui parasités gardent une croissance plus réduite. II y a donc interaction entre les variations climatiques et la présence actuelle du champignon.

De même, on observe une interaction entre la qualité de la station et les différences de croissance parasités-témoins : la croissance des arbres parasités est d'autant plus faible qu'ils se trouvent en station défavorable.

Ceci suggère deux hypothèses :

- soit l'installation du champignon est favorisée sur les arbres les plus sensibles aux événements climatiques, ou situés en conditions stationnelles défavorables,

- soit la présence du champignon provoque des pertes de croissance accrues durant les périodes climatiquement défavorables ou sur les mauvaises stations.

II est difficile de conclure car la date d'infestation par le champignon nous est inconnue. Cependant, selon Jacquiot et al. (1960, 1983), Phellinus robustus est un parasite peu actif qui affecte peu la vitalité de l'arbre. De plus, les différences de croissance que nous observons sont très anciennes. Enfin, les chênes montrent souvent une sensibilité aux perturbations environnementales particulièrement dépendante de facteurs prédisposants anciens (Becker et Lévy, 1983). La première hypothèse nous semble donc la plus probable.

Dans ce modèle où la prédisposition à l'infestation par le champignon, puis au creusement de la cavité, joue le rôle principal dans l'explication des différences de croissance observées, l'adéquation entre génotype - l'espèce dans notre cas - et environnement - le type de station - apparaît comme un des facteurs prédisposants majeurs.

Lorsque l'essence est adaptée à la station, la vigueur des arbres est élevée et n'est pas un facteur discriminant vis-à-vis de l'infestation par le champignon. C'est le cas du Chêne pédonculé dans la station $\mathrm{C}$, où les arbres sont très vigoureux ( $1,83 \mathrm{~mm}$ de largeur moyenne de cerne sur 150 ans) et où les différences de croissance entre arbres parasités et témoins ne sont pas significatives, jusqu'à une date récente tout au moins. Lorsque l'essence n'est pas adaptée à la station, la vigueur de l'arbre devient un facteur discriminant de l'infestation par le champignon.

Notre étude confirme que l'impact de la présence de Phellinus robustus sur la croissance en diamètre de la bille de pied est relativement faible en valeur absolue, jusqu'à 150 ans au moins. De 
plus, ce champignon se situe généralement dans les parties hautes de l'arbre - presque toujours à plus de $10 \mathrm{~m}$ du sol - , et le plus souvent sur des branches - dans $64 \%$ des cas étudiés (Dubreuil, 1996). Son impact économique est donc faible. Cependant, notre échantillon pourrait être biaisé par l'absence de chênes fortement et anciennement parasités, qui ont été probablement éliminés lors des coupes antérieures. Un grand nombre d'espèces animales sont potentiellement utilisatrices des cavités liées à la présence du champignon. Douze espèces d'oiseaux nicheurs cavernicoles ont ainsi été observées dans les parcelles étudiées. Ces cavités sont un élément important à prendre en compte dans une gestion orientée vers le maintien d'une diversité spécifique élevée.

Elvire HATSCH - J.-L. DUPOUEY
Équipe Phytoécologie
Unité d'Écophysiologie forestière
INRA
F-54280 CHAMPENOUX

\begin{tabular}{|c|}
\hline B. DUBREUIL $(\dagger)^{(1)}-$ J. GUILLAUD \\
Service départemental de la Moselle \\
OFFICE NATIONAL DES FORÊTS \\
3, boulevard Paixhans \\
F-57000 METZ \\
\hline
\end{tabular}

\section{BIBLIOGRAPHIE}

BECKER (M.), LÉVY (G.). - Le Dépérissement du Chêne. Les causes écologiques. Exemple de la forêt de Tronçais et premières conclusions. - Revue forestière française, vol. XXXV, $n^{\circ} 5,1983$, pp. 341-356.

DUBREUIL (B.). - La Cavité nidifiable en forêt domaniale de Hémilly (compte rendu 1995). Livret $n^{\circ} 3$ : Impact économique de la cavité et des pourritures qui favorisent son apparition, sur 3 triages du plateau lorrain. Metz : ONF Moselle, 1996. - 10 p. (document interne).

DUBREUIL (B.). - La Cavité nidifiable en forêt domaniale de Hémilly (compte rendu 1997). Livret $n^{\circ} 1$ : Description du cadre de l'étude. - Metz : ONF Moselle, 1997a. - 56 p. (document interne).

DUBREUIL (B.). - La Cavité nidifiable en forêt domaniale de Hémilly (compte rendu 1997). Livret $\mathrm{n}^{\circ} 2:$ Le genre Phellinus sur le chêne : écologie et relation avec la cavité nidifiable, parcelles 14,15 et 26 . - Metz: ONF Moselle, 1997b. - 21 p. (document interne).

DUPOUEY (J.-L.), BADEAU (V.). - Morphological variability of oaks (Quercus robur L., Quercus petraea (Matt.). Liebl., Quercus pubescens Willd.) in North-East of France. - Annales des Sciences forestières, vol. 50, $n^{\circ} 1$, 1993, pp. 35-40.

JACQUIOT (C.). - Contribution à l'étude de quelques espèces affines de la série des Igniaires. - Bulletin trimestriel de la société mycologique de France, vol. 76, n¹, 1960, pp. 83-106.

JACQUIOT (C.), ROBERT (D.), MOLLARD (A.). - Altération de la lignine du duramen de Chêne attaqué par le Polyporus sulfureus ((Bull), Fr), agent de pourriture cubique. In : Comptes-rendus du $108^{e}$ congrès national des sociétés savantes, Grenoble 1983, Section des Sciences. - Fascicule II, 1983, pp. 165-172.

PAUTZ (F.). - Origine et importance de la cavité arboricole pour les oiseaux. - Le Courrier de la Nature, 170, 1998, pp. 27-31.

PAUTZ (F.), DUBREUIL (B.). - Une étude décennale : la cavité dans l'arbre, 1994-2004. - Metz : ONF Moselle, 1997. - 20 p. (document interne).

SCHÉRER (J.-C.), FLEISCH (M.-R.). - Les Sécheresses 1996 et 1995 comparées à quelques sécheresses marquantes du siècle. - Les Cahiers du DSF (La santé des Forêts [France] en 1996), n 1, 1997, pp. 15-17.

(1) Bernard Dubreuil, qui a initié ce travail, est décédé en 1998. 


\section{IMPACT DU CHAMPIGNON PARASITE Phellinus robustus ET DES CAVITÉS NIDIFIABLES SUR LA CROIS- SANCE DES CHÊNES SESSILE ET PÉDONCULÉ (Résumé)}

Phellinus robustus Karst. est un champignon parasite qui attaque essentiellement le Chêne. Ce champignon provoque une pourriture fibreuse dans laquelle le Pic va creuser des cavités. Nous avons étudié l'influence des cavités et de ce champignon associé sur la croissance en diamètre de Chênes sessiles et pédonculés de façon à déterminer si la présence de ces défauts amène une perte de rendement au niveau forestier.

Les arbres parasités présentent une croissance radiale légèrement inférieure à celle des arbres témoins, souvent depuis une date très ancienne. Les différences de croissance sont plus importantes pour les arbres parasités situés hors de leur station optimale. Cependant, les pertes de croissance sont, dans l'ensemble, très faibles et l'impact économique minime. De plus, le maintien de ces arbres en peuplement permet de favoriser une biodiversité importante.

THE IMPACT OF THE FUNGAL PARASITE Phellinus robustus AND OF CAVITIES LIABLE TO BE USED AS NESTS ON THE GROWTH OF THE SESSILE AND PEDUNCULATE OAK (Abstract)

Phellinus robustus Karst is a fungal parasite whose primary target is the oak tree. This fungus causes fibrous rot in which woodpeckers dig hollows. We studied the influence of these cavities and this associated fungus on the radial growth of sessile and pedunculate oaks so as to determine whether such defects reduce the wood yield.

Radial growth of infected trees is slightly less than that of control trees, a phenomenon that can often be traced well into the past. The differences in growth are greater for trees that are not growing at their optimal site. However, growth losses are generally very small and the economic implications are minimal. Furthermore, maintaining these tree stands significantly contributes to biodiversity. 